\title{
Weitere Untersuchungen zur Entwicklung der Plastiden von Oenothera hookeri
}

(3. Mitteilung zur Entwicklungsgeschichte der Plastiden)

\author{
Von Wilhelm Menke
}

Aus dem Botanischen Institut der Universität zu Köln

(Z. Naturforschg. 15 b, 479-482 [1960]; eingegangen am 14. April 1960)

\begin{abstract}
An Hand von elektronenmikroskopischen Aufnahmen wird die Struktur der Plastiden im Sproßund Wurzelmeristem von Oenothera hookeri besprochen. Bei Fixierung der Gewebe mit Kaliumpermanganat werden die Membranen und die lamellaren Strukturen im Inneren der Plastiden so kontrastreich abgebildet, daß man die Morphogenese des Lamellen-Systems der Chloroplasten in seinen Einzelheiten verfolgen kann. Die Plastiden bestehen im Zustand ihrer größten entwicklungsgeschichtlichen Reduktion wahrscheinlich aus einem Stroma, welches von einer doppelten Membran umgeben ist. Die ersten Lamellen werden aus der inneren Plastidenmembran durch Einstülpung gebildet. So entstehen nach Ablösen der Einstülpungen von der Membran in sich geschlossene Doppellamellen, welche in kleinere Teilstücke zerfallen können. Diese teilen sich der Fläche nach, wofür ein möglicher Mechanismus, der ebenfalls auf Einstülpung beruht, angegeben wird. Die so entstandenen Lamellenstapel vereinigen sich nunmehr wahrscheinlich durch Vergrößerung einzelner Lamellen zu Schichten. Die Anordnung von Grana- und Stromalamellen in ausgewachsenen Chloroplasten wird durch ein Schema verdeutlicht.
\end{abstract}

In der 1 . Mitteilung ${ }^{1}$ dieser Reihe wurden Befunde an isolierten Chloroplasten veröffentlicht, die zeigten, daß Plastiden meristematischer Gewebe schon einen Feinbau besitzen können, der sich mit dem der Chloroplasten vergleichen läßt. Dieses Ergebnis konnte an Mikrotomschnitten durch Sproßvegetations-Punkte der Keimpflanzen von Oenothera hookeri bestätigt werden ${ }^{2}$. Für die Herstellung der Schnittpräparate wurden die Objekte mit Formalin fixiert und zur Kontrastierung mit Osmiumtetroxyd und Phosphorwolframsäure nachbehandelt. Bei diesem Verfahren werden auf den elektronenmikroskopischen Aufnahmen die korpuskularen Komponenten verhältnismäßig stark hervorgehoben, während die lamellaren Strukturen nur wenig in Erscheinung treten. Nun lassen sich, wie LuFT ${ }^{3}$ angegeben hat, die Membransysteme des Protoplasmas durch Fixierung mit Kaliumpermanganat kontrastreich darstellen. So lag es nahe, zu versuchen, mit dieser $\mathrm{Me}$ thode auch die lamellaren Strukturen in den Plastiden meristematischer Gewebe deutlicher nachzuweisen, als das bisher möglich war.

\section{Material und Methoden}

Die Anzucht der Keimpflanzen erfolgte in der früher angegebenen Weise ${ }^{2}$. Außer Keimlingen wurden auch Vegetationspunkte und Wurzelspitzen von Pflanzen im Rosettenstadium untersucht. Die Fixierung wurde nach den Angaben von $\mathrm{LuFr}^{3}$ mit 1-proz. wäßriger Kaliumpermanganatlösung $\left(p_{\mathrm{H}}=7\right)$ vorgenommen. Die Poly- merisation des Methacrylatgemisches erfolgte hier im Thermostaten bei $65^{\circ} \mathrm{C}$. Da auf eine eindeutige Lokalisierung der Zellen im Gewebe Wert gelegt wurde, war die Herstellung ausgedehnter Schnitte erforderlich. Diese wurde durch Verwendung unseres Eigenbaumikrotoms, welches eine kontinuierliche Regulierung der Schneidegeschwindigkeit zuläßt, erleichtert.

\section{Die Plastiden des Sproßmeristems}

Im Sproßscheitel der Keimpflanzen von Oenothera hookeri sind die typisch meristematischen Zellen mit kleinen Vakuolen im wesentlichen auf die Blattanlagen beschränkt, während zu Rosetten herangewachsene Pflanzen ein ausgedehntes Sproßmeristem enthalten. In Abb. $1^{*}$ ist ein Längsschnitt durch die erste Blattanlage einer Keimpflanze wiedergegeben. Diese Übersichtsaufnahme zeigt fast die gesamte Blattanlage. Die Zellen mit großen Vakuolen in der unteren linken Ecke des Bildes gehören schon der Übergangszone zwischen Blattanlage und Sproßachse an. Selbst bei dieser verhältnismäßig schwachen Vergrößerung lassen sich die Plastiden von den anderen Zellbestandteilen eindeutig unterscheiden. Infolge der starken Kontrastierung der Membranen und der lamellaren Strukturen erscheinen die Plastiden gegen das Cytoplasma klar abgegrenzt und ihr Schichtenbau ist deutlich zu erkennen. Jede Plastide enthält

1 W. MENKE, Z. Naturforschg. 11 b, 215 [1956].

2 W. Menke, Z. Naturforschg. 14 b, 394 [1959].

3 J. A. Lurt, J. Biophys. Biochem. Cytol. 2, 799 [1956].

* Abb. 1-7 s. Tafel S. 482 a, b, c. 
im schwächer kontrastierten Stroma wenigstens eine, meistens aber mehrere Schichten. Die Mitochondrien sind viel kleiner als die Plastiden und eben zu erkennen. Der feinere Bau der Schichten ist aus der stärker vergrößerten $\mathrm{Abb} .2$ zu ersehen, welche einen Teil einer Zelle aus der Blattanlage einer Keimpflanze wiedergibt. Diese Blattanlage hatte etwa die gleiche Größe wie die in Abb. 1 gezeigte. Die abgebildete Zelle ist die vierte, von der Blattspitze gerechnet, und liegt mitten im Blattgewebe. Man erkennt, daß die Schichten Aggregationen von größeren und kleineren in sich geschlossenen Doppellamellen sind. Bemerkenswert ist, daß die kleineren Lamellen häufig in Stapeln übereinanderliegen und daß diese manchmal von größeren Doppellamellen durchzogen werden. Bei dieser Verteilung der Doppellamellen wird man geneigt sein, anzunehmen, daß die kleineren den Granalamellen, die größeren den Stromalamellen der vollentwickelten Chloroplasten entsprechen. Es sei aber darauf hingewiesen, daß sich die Schichtenstruktur in diesem Stadium noch im Aufbau befindet und es noch nicht feststeht, ob sich die Grana der vollentwickelten Chloroplasten direkt aus den Stapeln von kleineren Lamellen entwickeln. Dies braucht um so weniger der Fall zu sein, als beim Wechsel der Außenbedingungen die Granastruktur der Chloroplasten anscheinend verschwinden und wieder entstehen kann. Außer diesen Aggregationen von Doppellamellen liegen, im Stroma verteilt, noch einzelne Doppellamellen verschiedener Größe (Abb. 2, 3,5). In der Plastide am oberen Bildrand von Abb. 2 steht eine solche Lamelle mit der Plastidenmembran in Verbindung. Bei stärkerer Vergrößerung erkennt man, daß die Plastidenmembran doppelt konturiert ist (Abb.4), und die mit ihr zusammenhängenden Lamellen erscheinen als Ausstülpung der inneren Kontur. Außerdem entspringen der Plastidenmembran gelegentlich Tubuli mit kreisförmigem Querschnitt. Die Unterbrechungen der Plastidenmembran sind wenigstens teilweise Artefakte. Es wäre jedoch von großem Interesse, zu wissen, ob die Plastidenmembran wie die Kernmembran zu gewissen Zeiten von Poren durchbrochen ist, welche den Durchtritt hochmolekularer Verbindungen erleichtern. Die Plastiden im Sproßmeristem von Pflanzen im Rosettenstadium sind einfacher gebaut. Sie enthalten im Stroma nur einzelne Lamellen und entsprechen hinsichtlich ihrer Entwicklung etwa den unten beschriebenen Plastiden des Wurzelmeristems.

\section{Der feinere Bau der Schichten in ausgewachsenen Chloroplasten}

Trotz aller Bemühungen war es bisher nicht möglich, den lamellaren Feinbau der Schichten, insbesondere den Zusammenhang zwischen Grana- und Stromalamellen aufzuklären. Als Bauelemente der Schichten sind die beim Zerfall von isolierten Chloroplasten freiwerdenden Grana- und Stromalamellen anzusehen ${ }^{4}$, die als in sich geschlossene, aus Proteinen und Lipiden bestehende Doppellamellen aufzufassen sind. Die sich entwickelnden Plastiden der Blattanlagen zeigen nun die Anordnung der Lamellen viel deutlicher als ausgewachsene Chloroplasten

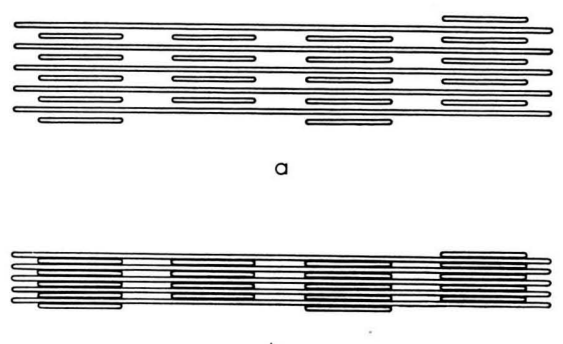

b

Abb. 8. Bauprinzip einer Schicht in einem ausgewachsenen Chloroplasten. a) Anordnung der Grana- und Stromalamellen. b) Durch Zusammenrücken der Lamellen entsteht ein Bild, wie es Querschnitte durch Chloroplasten im Elektronenmikroskop zeigen.

und daher gelingt es, die weniger übersichtliche Struktur der Schichten in ausgewachsenen Chloroplasten aus diesen abzuleiten. In Abb. 8 ist das Bauprinzip einer Schicht in schematischer Form wiedergegeben. Die Anordnung von Grana- und Stromalamellen ist zunächst aus Abb. 8 a zu ersehen. Bei dichter Packung entsteht ein Bild (Abb. 8 b), wie es Querschnitte durch Chloroplasten bei starker Vergrößerung im Elektronenmikroskop zeigen. Die Zahl der Granalamellen ist scheinbar gleich der Zahl der Stromalamellen und die Granalamellen erscheinen dicker als die Stromalamellen. In Wirklichkeit ist bei gleicher Zahl und regelmäßigem Wechsel von Granaund Stromalamellen die Zahl der Einzellamellen in den Grana doppelt so groß wie in den dazwischen liegenden Stromalamellen, und die Lamellen in den Grana erscheinen dicker, weil sie aus einer Granaund einer Stromalamelle aufgebaut sind. Über den molekularen Feinbau der Lamellen wird demnächst in anderem Zusammenhang berichtet werden.

4 W. Menke, Protoplasma 35, 115 [1940]. 


\section{Die Plastiden des Wurzelmeristems}

Die Plastiden in den Initialen des Meristems von Keimwurzeln sind nur wenig kleiner als die Plastiden in jungen Blattanlagen. Sie sind wie diese von den Mitochondrien eindeutig unterscheidbar. Sie werden ebenfalls von einer doppelt konturierten Membran begrenzt. Jedoch enthalten sie keine Schichten und Lamellenstapel. Wohl sieht man in mehr als der Hälfte der Plastidenschnitte eine, gelegentlich auch mehrere isoliert liegende Doppellamellen verschiedener Ausdehnung. Diese laufen häufig entlang der Plastidenmembran und können bei schwacher Vergrößerung leicht übersehen werden. Gelegentlich findet man auch hier Lamellen, die von der inneren Kontur der Plastidenmembran ausgehen. Außerdem kommen die schon beschriebenen Tubuli vor, die einzeln oder in Gruppen der Plastidenmembran entspringen. Etwas vielgestaltiger sind die Plastiden in Wurzelspitzen von Pflanzen im Rosettenstadium, wo neben solchen mit kreisförmigem oder elliptischem Querschnitt (Abb. 6,7) sich häufig amoeboid geformte finden. Strukturell unterscheiden sie sich von Plastiden in Keimwurzeln nicht wesentlich, obwohl diese auf Filtrierpapier bei normalem Licht-Dunkelwechsel angezogen worden waren, während die Pflanzen im Rosettenstadium auf Erde gewachsen waren, wobei die Wurzeln sich also im Dunkeln befanden. In Plastiden aus Wurzeln von Pflanzen im Rosettenstadium sind die Schnitte durch Doppellamellen nicht selten ringförmig geschlossen. Die Plastiden in Wurzelspitzen anderer Pflanzen weisen offenbar einen sehr ähnlichen Bau auf ${ }^{5}$.

Die Unterschiede zwischen den Plastiden der Blattanlagen und den Plastiden der Wurzelspitzen hängen natürlich mit der Bildung der für die Photosynthese notwendigen Strukturen zusammen. Die Plastiden der Blattanlagen sind in Entwicklung begriffene Chloroplasten, die der Wurzelspitze Leukoplasten.

\section{Zur Morphogenese der Lamellarstruktur}

In der vorhergehenden Mitteilung ${ }^{2}$ wurden einige Vermutungen über die Morphogenese der Plastidenstruktur geäußert, wobei biochemische Gesichtspunkte im Vordergrund standen. Im folgenden soll

5 Allium cepa und Allium sativum: K. R. Porter, u. P. D. Machado, J. Biophys. Biochem. Cytol. 7, 167 [1960]; Zea mays und Vicia faba: E. Hertz, Z. Naturforschg. 12 b, 283 auf die Entwicklung der Lamellarstruktur der Chloroplasten vom morphologischen Standpunkt aus eingegangen werden.

Die Frage, welchen Bau die Plastiden im Zustand ihrer größten entwicklungsgeschichtlichen Reduktion besitzen, ist noch nicht eindeutig geklärt. Nach Mühlethaler und Frey-Wyssling ${ }^{6}$ bestehen sie aus einem nicht strukturierten Stroma, das von einer Doppelmembran begrenzt wird. Ihre Größe soll nur etwa $0,02 \mu \mathrm{im}$ Durchmesser betragen. Die kleinsten bisher bei Oenothera aufgefundenen Plastiden besitzen demgegenüber einen Durchmesser von der Größenordnung $1 \mu$. Den Befund, daß die Mehrzahl der Schnitte durch Plastiden des Wurzelmeristems eine Doppellamelle enthält, kann man so deuten, daß entweder nicht alle Plastiden dieses Strukturelement besitzen, oder daß alle Plastiden eine Lamelle enthalten, die jedoch wegen ihrer begrenzten Ausdehnung von einem Teil der Schnitte nicht getroffen ist. Falls es bei Oenothera Plastiden ohne Lamelle gibt, so sprechen die mitgeteilten Befunde dafür, daß die ersten Lamellen durch Hineinwachsen der inneren Plastidenmembran in das Stroma gebildet werden, wie das kürzlich MüHLETHALER und FrEY-WyssLing $^{6}$ für die Plastiden anderer Pflanzen beschrieben haben. Wenn die ersten Lamellen jedoch aus der inneren Membran herauswachsen und nicht aus dem Stroma neugebildet werden, so muß man mit der Möglichkeit rechnen, daß die Lamellen überhaupt nicht neu gebildet werden können, sondern immer aus ihresgleichen entstehen; denn es ist nicht einzusehen, warum die ersten Lamellen aus der inneren Plastidenmembran herauswachsen, die späteren jedoch aus dem Stroma neu gebildet werden. Nun hat es aber nicht den Anschein, als ob alle Lamellen aus der inneren Plastidenmembran herauswüchsen, und so drängt sich die Frage auf, wie die Lamellenstapel aus einer Lamelle entstehen. In den in Abb. 3 und 4 wiedergegebenen Aufnahmen sieht man an den mit Pfeilen bezeichneten Stellen Strukturen, die man so auffassen kann, daß von der Kante, an einer Stelle auch von der Seite einer Doppellamelle her eine Einstülpung in diese hineinwächst. Wenn diese Deutung richtig ist, so ergibt sich daraus zwanglos ein Mechanismus für die Teilung der Doppellamellen in Richtung der Fläche-

[1957]; Zea mays: H. H. Mollenhauer, J. Biophys. Biochem. Cytol. 6, 431 [1959].

6 K. Mühlethaler u. A. Frey-Wyssling, J. Biophys. Biochem. Cytol. 6, 507 [1959]. 

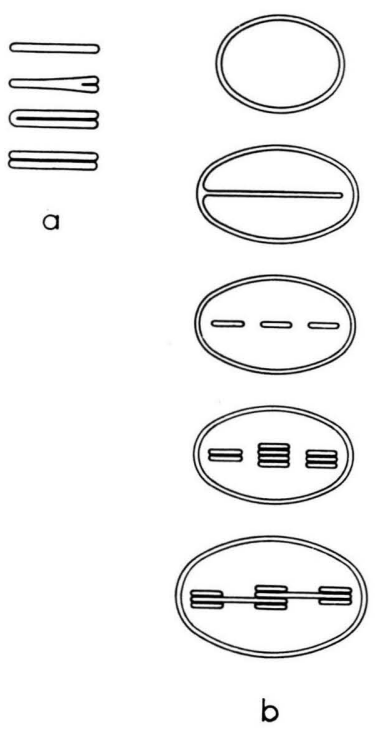

Abb. 9. a) Teilung von Doppellamellen durch Einstülpung von der Kante her. b) Vereinfachtes Schema zur Entwicklung der Schichtenstruktur.

normalen und für das Entstehen von Lamellenstapeln. Dieser ist in Abb. 9 schematisch wiedergegeben. Die aufeinanderfolgenden Vorgänge, die zur Ausbildung von Lamellenstapeln führen, sind: Auswachsen der inneren Membranschicht ins Stroma, Zerfall der so entstandenen Doppellamelle und Teilung der einzelnen Teilstücke der Fläche nach; diese wird durch Einstülpung der Lamellen von der Kante her eingeleitet. Man kann sich natürlich auch vorstellen, daß nicht erst ein Zerfall der zuerst gebildeten Lamellen in Teilstücke eintritt, sondern daß sich diese nach dem angegebenen Mechanismus verdoppeln und erst später zerfallen. Dann sollten jedoch alle in einer Ebene nebeneinanderliegenden Lamellenstapel die gleiche Zahl von Lamellen enthalten, was im allgemeinen nicht der Fall ist. Es bleibt noch zu klären, wie aus den in einer Ebene liegenden Lamellenstapeln eine Schicht entsteht, woher also die Stromalamellen stammen. Hier liegt es nahe, anzunehmen, daß diese durch Wachstum einzelner Lamellen sich zunächst vergrößern und dann entweder durch benachbarte Stapel hindurchwachsen oder sich mit anderen Lamellen vereinigen. Eine Vereinigung von kleineren Doppellamellen zu größeren war schon von Hodge $^{7}$ und Mitarbb. vermutet worden. Dieses Entwicklungsschema erklärt zwanglos, warum die kleineren Lamellen und Lamellenstapel häufig in einer Ebene liegen, und warum in den Grana die Lamellen in Stapeln übereinander angeordnet sind. Es darf jedoch nicht übersehen werden, daß besonders der angegebene Teilungsmechanismus noch durch weitere Untersuchungen gesichert werden muß, da hier eine Täuschung durch Artefakte nicht von der Hand zu weisen ist. Eine andere nicht auszuschließende Möglichkeit für die Entstehung von Lamellenstapeln wäre noch die, daß sich zunächst isoliert liegende Doppellamellen später zu Stapeln zusammenlagern.

Wenn diese Überlegungen die Morphogenese der Chloroplastenstruktur in den Grundzügen zutreffend wiedergeben, so folgt daraus, daß die innere Plastidenmembran dem Lamellarsystem der Chloroplasten homolog ist. Wenn ferner das ganze Lamellarsystem der Chloroplasten aus der inneren Plastidenmembran durch Wachstum, Zerfall und Teilung entsteht, so liegt es nahe, in der inneren Plastidenmembran eine idiotypische Komponente der Plastiden im $\mathrm{Zu}$ stand ihrer stärksten entwicklungsgeschichtlichen Reduktion zu sehen oder sie als Träger von idiotypischen Komponenten aufzufassen. Durch die Lokalisation dieser idiotypischen Komponenten in der Membran wäre die Gewähr gegeben, daß bei der Plastidenteilung jede Tochterplastide mit Sicherheit einen Teil dieses Membrankomplexes mitbekommt. Die Frage nach der Natur der idiotypischen Komponenten der Plastiden und ihre Bedeutung für die Plastidenvererbung wurde erst vor kurzem diskutiert $^{8}$, so daß sich an dieser Stelle ein Eingehen darauf erübrigt.

Die vorliegende Arbeit wurde mit Mitteln der D e u t . schen Forschung gem e in schaf t ausgeführt. Fräulein Helga Lehmans bin ich für ihre Mitarbeit besonderen Dank schuldig.

\footnotetext{
7 A. J. Hodge, J. D. McLean u. F. V. Mercer, J. Biophys. Biochem. Cytol. 2, 597 [1956].

8 W. Menke, Z. Vererb.-Lehre, im Druck.
} 
W. Menke, Weitere Untersuchungen zur Entwicklung der Plastiden von Oenothera hookeri (S. 479)

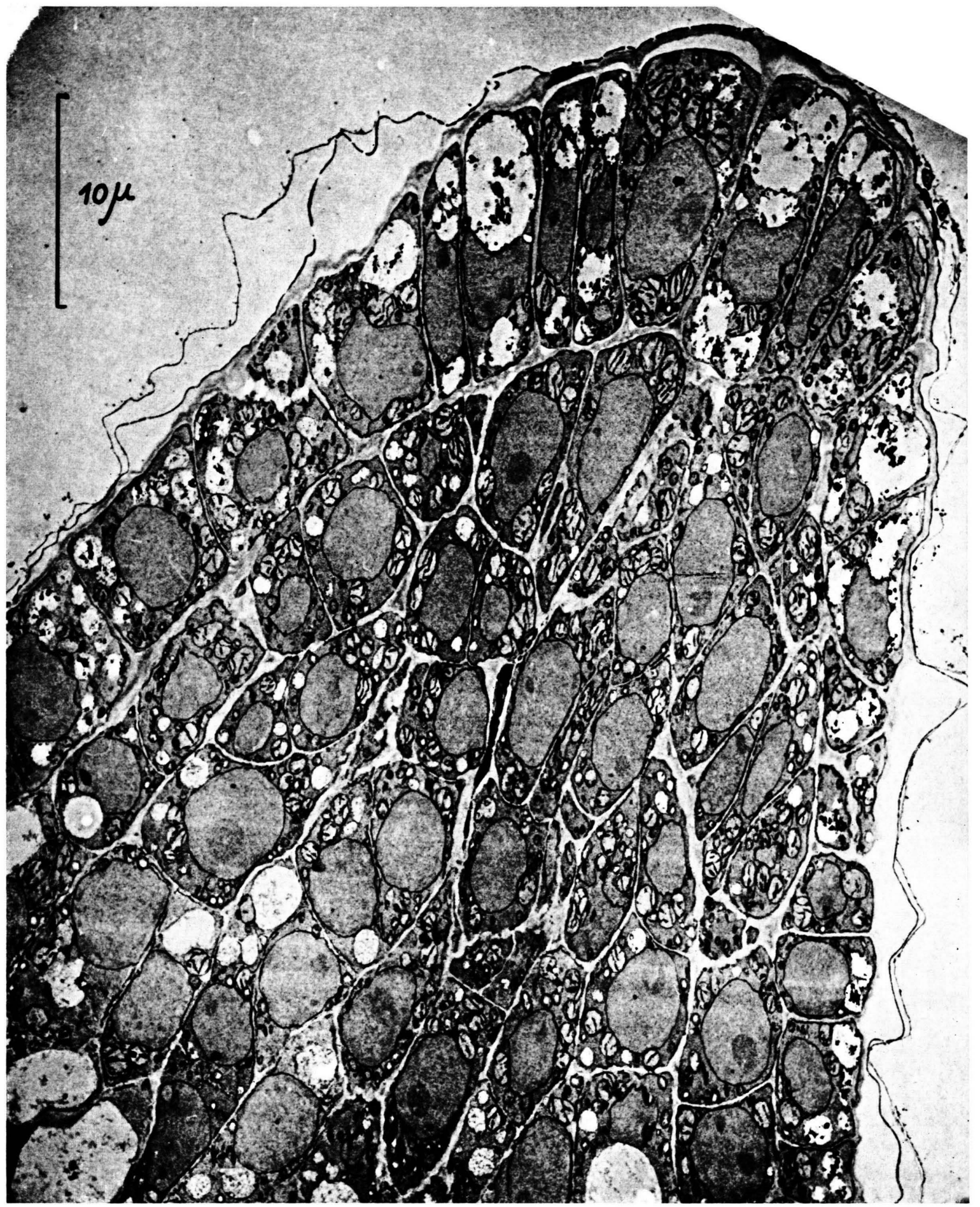

Abb. 1. Längsschnitt durch die erste Blattanlage einer Keimpflanze. 


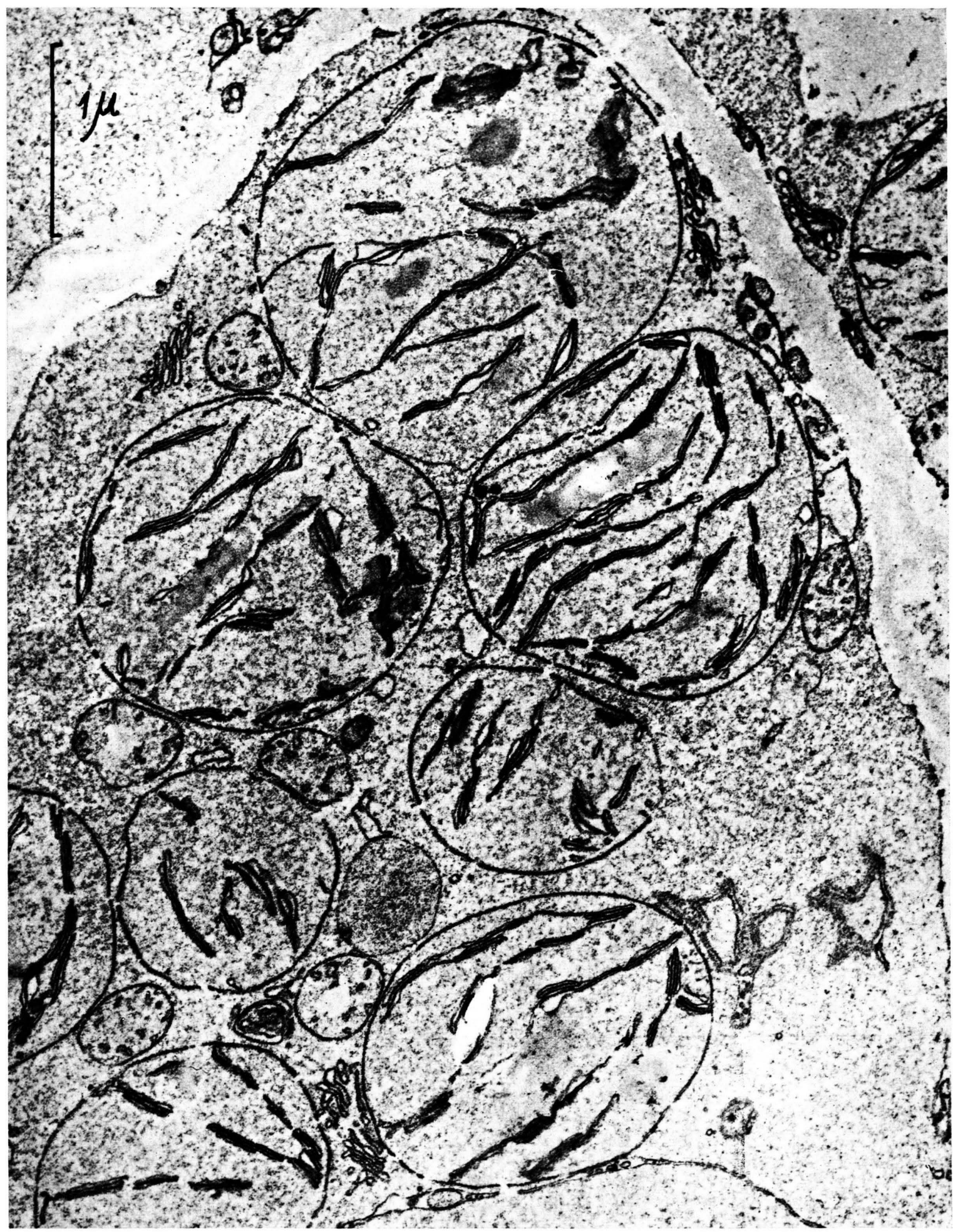

Abb. 2. Teil einer Zelle aus einer Blattanlage von etwa derselben Größe wie die in Abb. 1 gezeigte. 


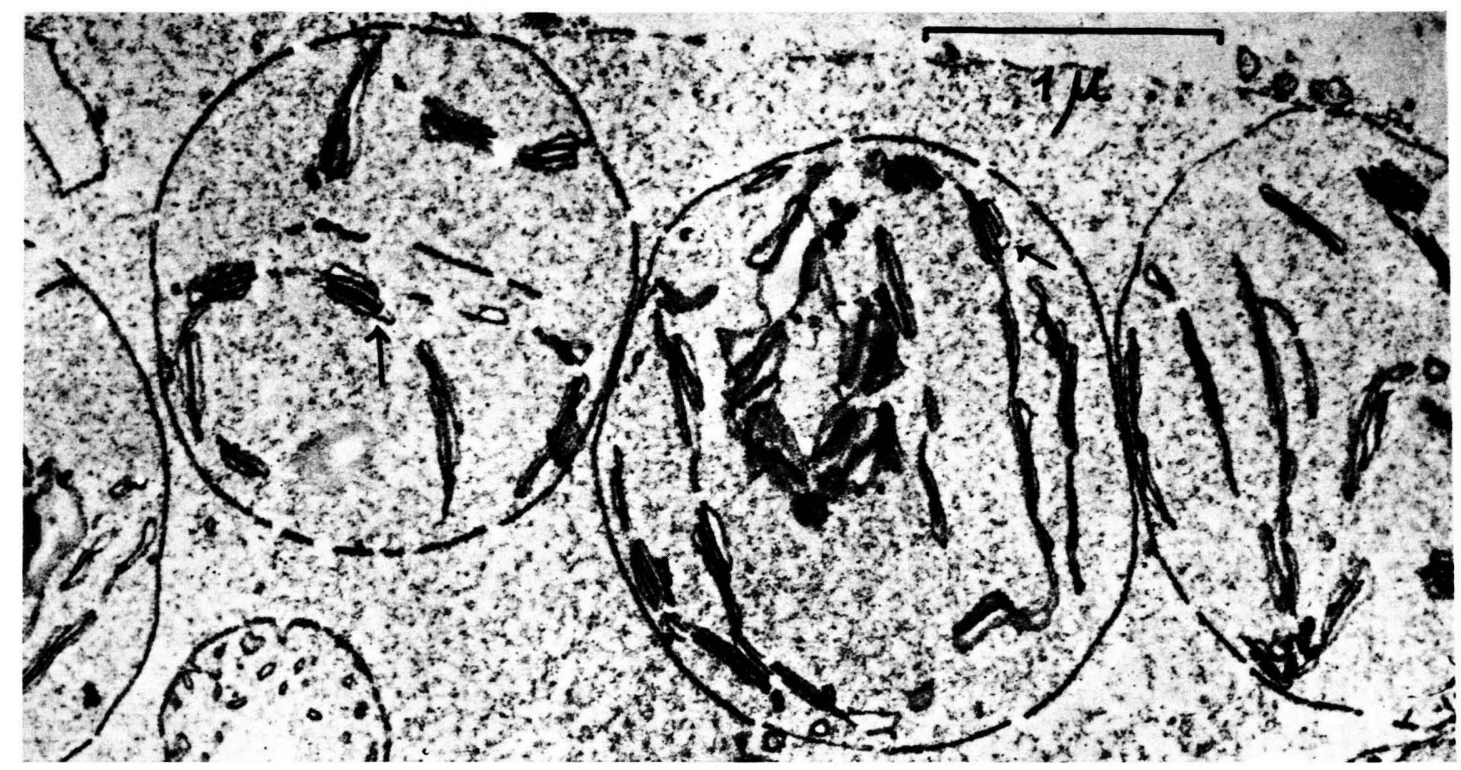

Abb. 3. Teil einer Zelle wie in Abb. 2. An den durch Pfeilen gekennzeichneten Stellen vermutlich in Teilung begriffene Lamellen.

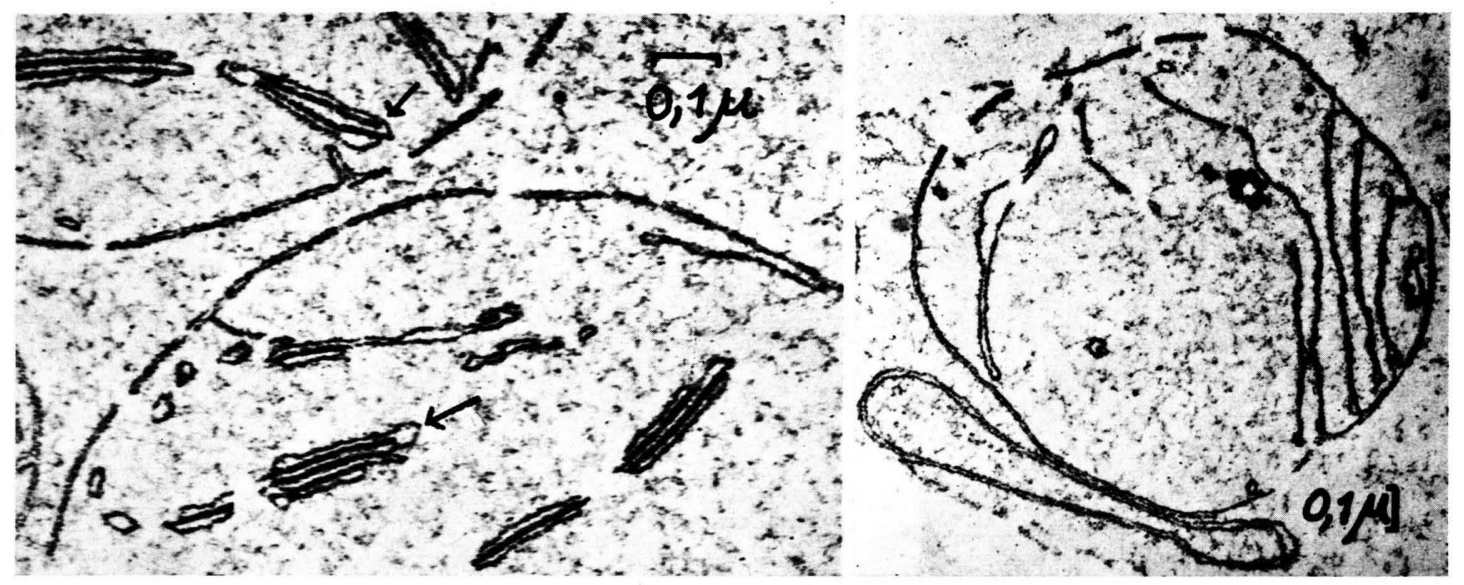

Abb. 4. Stärker vergrößerter Ausschnitt aus einer Zelle wie Abb. 5. Plastide mit einzelnen Doppellamellen aus einer in Abb. 2 und 3.

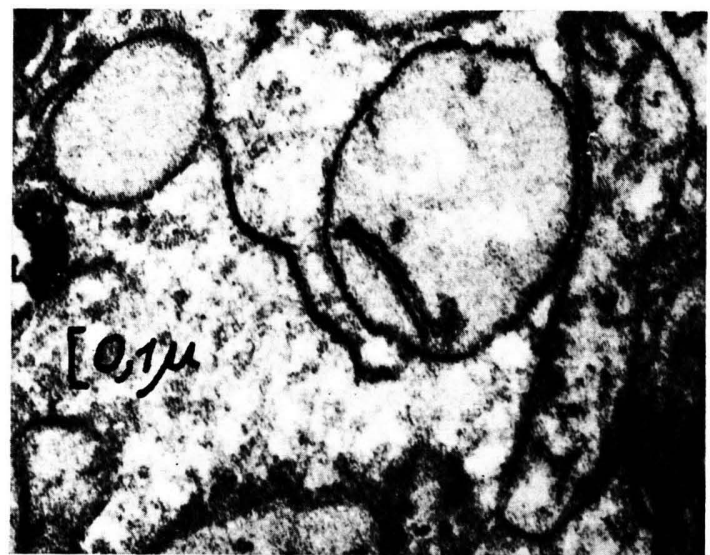

Abb. 6. 2 Plastiden und ein Mitochondrion aus einer Rhizodermiszelle, die eben noch im Bereich der Calyptra liegt. Epidermiszelle von der Spitze einer Blattanlage.

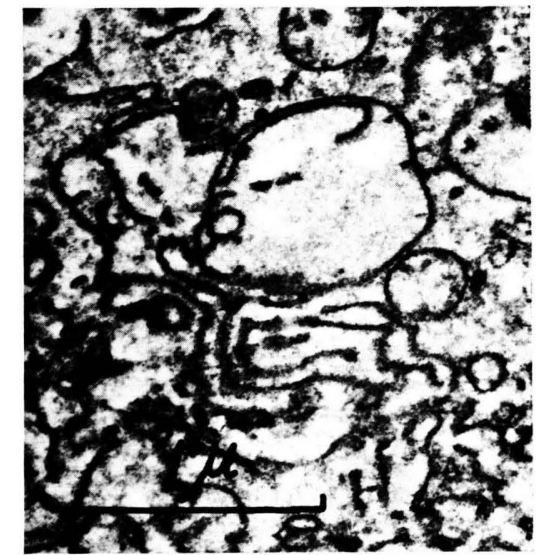

Abb. 7. Plastide aus einer Rindenzelle im Bereich der Calyptra.

Zeitschrift für Naturforschung 15 b, Seite 482 c. 
W. Kreutz und W. Menke, Strukturuntersuchungen an Plastiden (S. 483)

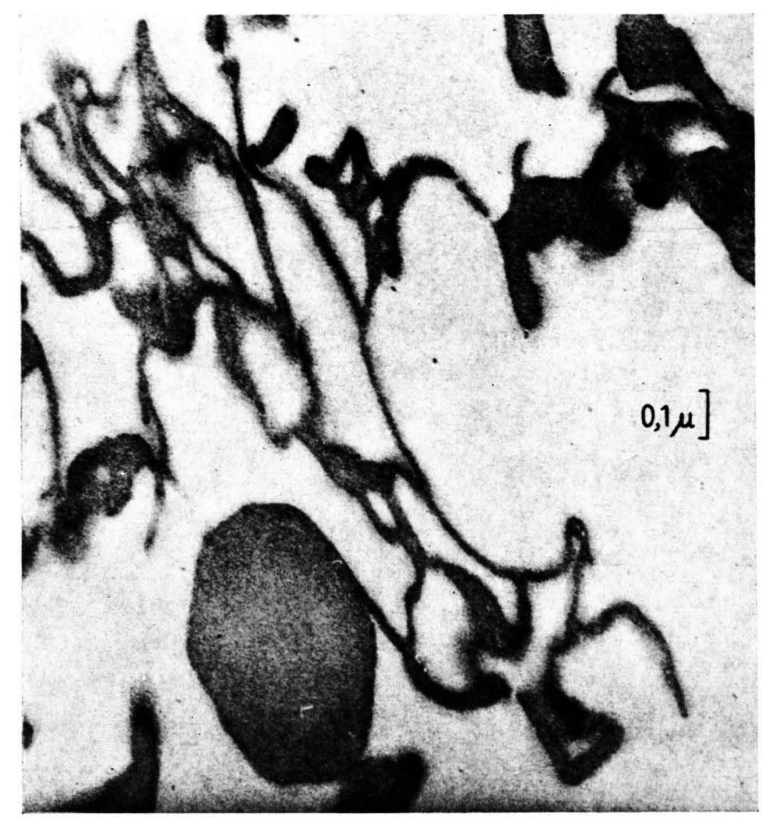

Abb. 4 a.

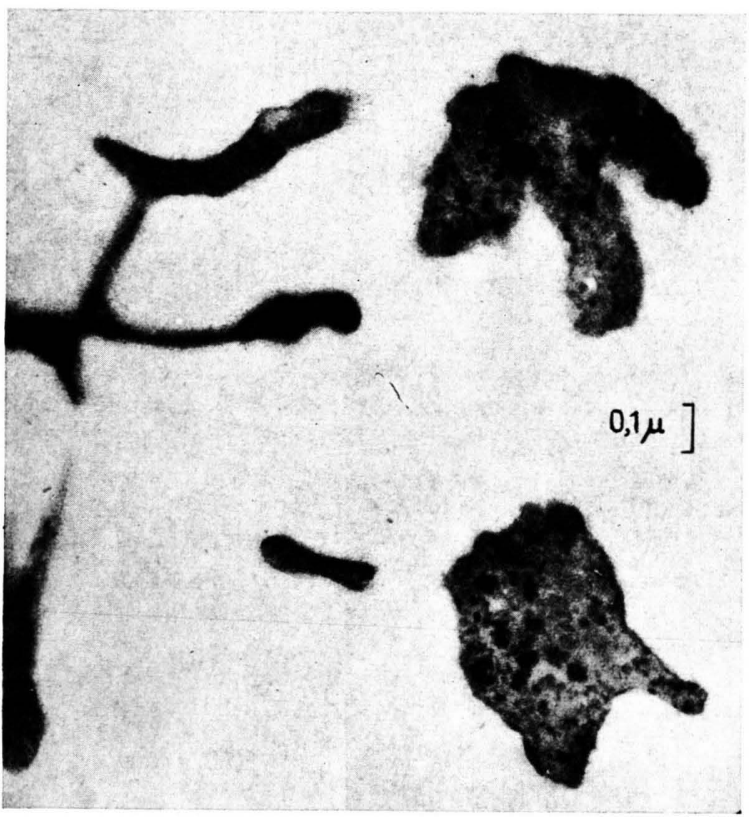

Abb. 4 b.

Abb. 4. Schnitte durch gefriergetrocknete, isolierte Chloroplasten von Antirrhinum majus. a) lipidfrei (vor der Fixierung extrahiert). b) lipidhaltig. Bei der Einbettung in Methacrylat erfolgte ein weitgehender Zerfall der Chloroplasten. 